\title{
Influence of resting lung diffusion on exercise capacity in patients with COPD
}

Mehrdad Behnia ${ }^{1,4^{*}}$, Courtney Wheatley ${ }^{2}$, Alberto Avolio $^{3}$ and Bruce Johnson ${ }^{2}$

\begin{abstract}
Background: Lung diffusing capacity for carbon monoxide (DLCO) gives an overall assessment of functional lung surface area for gas exchange and can be assessed using various methods. DLCO is an important factor in exercise intolerance in patients with chronic obstructive pulmonary disease (COPD). We investigated if the intra-breath (IBDLCO) method may give a more sensitive measure of available gas exchange surface area than the more typical single breath (SBDLCO) method and if COPD subjects with the largest resting DLCO relative to pulmonary blood flow (Qc) would have a more preserved exercise capacity.

Methods: Informed consent, hemoglobin, spirometry, SBDLCO, IBDLCO, and QC during IBDLCO were performed in moderate to severe COPD patients, followed by progressive cycle ergometry to exhaustion with measures of oxygen saturation $\left(\mathrm{SaO}_{2}\right)$ and expired gases.

Results: Thirty two subjects (47\% female, age $66 \pm 9$ yrs., BMl $30.4 \pm 6.3 \mathrm{~kg} / \mathrm{m}^{2}$, smoking hx $35 \pm 29$ pkyrs, $2.3 \pm 0.8$ on the 0-4 GOLD classification scale) participated. The majority used multiple inhaled medications and $20 \%$ were on oral steroids. Averages were: FEV $1 /$ FVC $58 \pm 10 \%$ Pred, peak $V_{2} 11.4 \pm 3.1 \mathrm{ml} / \mathrm{kg} / \mathrm{min}$, and IBDLCO $72 \%$ of the SBDLCO ( $r=0.88$, SB vs IB methods). Using univariate regression, both the SB and IBDLCO (\% predicted but not absolute) were predictive of $\mathrm{VO}_{2}$ peak in $\mathrm{ml} / \mathrm{kg} / \mathrm{min}$; SBDLCO/Qc $(r=0.63, p<0.001)$ was the best predictor of $\mathrm{VO}_{2}$ peak; maximal expiratory flows over the mid to lower lung volumes were the most significantly predictive spirometric measure $(r=0$. $49, p<0.01)$. However, in multivariate models only BMI added additional predictive value to the SBDLCO/Qc for predicting aerobic capacity $(r=0.73)$. Adjusting for current smoking status and gender did not significantly change the primary results.
\end{abstract}

Conclusion: In patients with moderate to severe COPD, preservation of lung gas exchange surface area as assessed using the resting SBDLCO/Qc appears to be a better predictor of exercise capacity than more classic measures of lung mechanics.

Keywords: Lung surface area, Gas exchange, Dyspnea, Gas transfer

\section{Background}

Causes contributing to exercise limitation in patients with chronic obstructive pulmonary disease (COPD) are complex [1-3]. Previous studies have suggested that while lung mechanics clearly play an important role, there are many other factors that contribute to this limitation such as heterogeneity of the disease process, lifestyle issues, such as weight and activity patterns, deconditioning, disease-related inflammatory processes,

\footnotetext{
* Correspondence: doctorbehnia@gmail.com

${ }^{1}$ University of Central Florida School of Medicine and Division of Critical Care, Florida Hospital, Orlando, FL, USA

${ }^{4}$ PO Box 953814, Lake Mary, FL 32795, USA

Full list of author information is available at the end of the article
}

perception, as well as associated comorbidities such as cardiovascular disease [4-6]. Pulmonary function measures representing the degree of obstruction and severity of hyperinflation (e.g., inspiratory capacity or IC) appear important as well as less appreciated factors such as a blunted cardiac output, either due to airway obstruction and rise in intra-thoracic pressure or from the development of pulmonary hypertension [7-9]. As a result, exercise capacity as a whole has been used as a prognostic indicator in the COPD population and as such is a good assessment of the integrative factors involved in the disease [10]. In addition, as stated by the GOLD initiative (Global Initiative for Chronic 
Obstructive Lung Disease classification for air flow obstruction), improvement in exercise tolerance is recognized as an important goal of COPD treatment.

From the lung volume reduction surgery data, it has also been found that certain patterns of disease and perhaps more severe emphysema may be associated with worse exercise tolerance $[11,12]$. Of the common relatively simple screening tests, a low lung diffusing capacity for carbon monoxide (DLCO) has been shown to not only suggest a more emphysematous pathophysiology but has also been a predictor of exercise capacity and in particular exercise induced oxygen desaturation $[1,13]$. There are different ways to quantify DLCO, from the typical single breath method (SBDLCO), to various rebreathe, steady state, open-circuit and intra-breath techniques [14]. While the latter methods may represent in some sense more physiological quantification of functional lung surface area for gas transfer or exchange, the single breath method has been standardized with wellestablished predictive norms for clinical use [15].

The intra-breath method (IBDLCO) is interesting in that it potentially represents a relatively simple way to quantify DLCO in patients that may struggle with longer breath hold times and there is potential for use during exercise to quantify alveolar-capillary surface area recruitment. It requires the exhalation of test gas typically near residual volume, followed by a deep inhalation and essentially instantaneous exhalation back towards residual volume (RV). The expiratory sampling relies on a fast response $\mathrm{CO}$ analyzer and as exhalation continues towards RV, the DLCO at any point is dependent then on the exhaled lung volume and each time point would represent a DLCO that is a mix of $\mathrm{CO}$ uptake and mixing with other lung gases $[16,17]$. An advantage of this technique compared to other more discrete techniques is that the exhaled gas stream is used in its entirety to calculate DLCO.

In patients with lung disease it is likely that the intrabreath method may be more sensitive to disease pathology relative to the single breath method due to abnormalities in ventilation and perfusion and delayed time constants for ventilation with the shortened gas exchange times.

Lung diffusion is dependent on pulmonary capillary blood volume $(\mathrm{Vc})$ and alveolar-capillary gas exchange surface area, usually reported as membrane diffusion capacity. While these components of DLCO can be estimated by performing DLCO at multiple oxygen concentrations or with a second gas such as nitric oxide (DLNO), a surrogate may be obtained for blood volume by examining the DLCO relative to cardiac output $(\mathrm{Qc})$. Since a rise in $\mathrm{Q}$ tends to be the major reason for distension or recruitment of capillaries, a larger ratio would be indicative of a healthier phenotype.

Thus in the present study we were interested in the role of resting DLCO in predicting exercise capacity in a relative diverse group of COPD patients. More specifically we were interested if a higher intra-breath to single breath DLCO ratio or a higher DLCO relative to Qc ratio would better predict exercise capacity relative to other common measures of lung mechanics. We hypothesized that those subjects with a higher IBDLCO relative to SBDLCO or a higher SBDLCO/Qc ratio would have better preserved exercise capacity.

\section{Methods}

\section{Ethics and consent}

The study, ethics, and consent forms were reviewed and approved by the Western Institutional Review Board (WIRB, study number 1153374).

\section{Subjects}

Patients with a history of COPD that were sent for clinical pulmonary function testing and/or exercise testing were offered enrollment. Inclusion criteria included established patients with a history of COPD, on stable medications without recent exacerbation (within 3 months). Exclusion criteria included, oxygen dependence an inability to exercise and/or a BMI $>42$. Both past and current smokers were allowed to participate with 7 of the participants being current smokers. Prior to participation, the study goals and requirements were reviewed with the patients. If willing to participate, patients signed informed consent.

\section{Overview of study}

After reporting to the outpatient clinic, study participants filled out the St. George's Respiratory quality of life questionnaire (SGQOL), performed pulmonary function testing (PFTs) which included resting measures of maximal lung volumes and flow rates using classical spirometry. In addition the assessment of lung diffusing capacity for carbon monoxide (DLCO) was obtained using the classical single breath $(\mathrm{SB})$ technique and was also obtained using the intra-breath method (IB) which included a measure of pulmonary blood flow (Qc). A small blood sample was obtained prior to testing for assessment of hemoglobin in order to correct the measure of DLCO. Subjects subsequently performed cardiopulmonary exercise testing (CPET) using the CareFusion Vmax Encore metabolic cart (San Diego, CA) with a Corival recumbent cycle ergometer (Lode, Netherlands). The test protocol started with 20 watts for both men and women and increased by 10 watts every $2 \mathrm{~min}$. Prior to exercise testing, subjects were instrumented with a 12 lead ECG, and a forehead pulse oximeter for peripheral oxygen saturation $\left(\mathrm{SaO}_{2}\right)$ for continuous monitoring. Subjects wore a nose clip and breathed on a mouthpiece for continuous measurement of gas exchange during the exercise test. During the last $30 \mathrm{~s}$ of each workload, a 12 lead ECG recording was printed, blood pressure (BP) assessed, perceived dyspnea score (010 scale) and perceived exertion (an assessment of total 
body effort) was rated by subjects, and an average of the $\mathrm{HR}$ and $\mathrm{SpO}_{2}$ over this period was determined. The goal was to obtain at least 2-3 work levels for each subject. Subjects were encouraged to exercise to near exhaustion based on symptom limitation by achieving an RPE of 1718 on the Borg 6-20 scale or a dyspnea score $\geq 7$ on the 0 10 score [18]. Upon reaching peak symptom limited exercise, subjects performed active recovery where they continued to pedal with no resistance and remained on the mouthpiece for $1 \mathrm{~min}$. After this the subject stopped pedaling and was given time for $\mathrm{HR}$ and BP to return to baseline before being dismissed.

\section{Pulmonary function and single breath DLCO}

Spirometry was performed using pneumotachographbased pulmonary function equipment that has passed evaluation using 24-wavefroms recommended by the American Thoracic Society (ATS). Classic single breath DLCO was determined using a commercial instrument that utilizes a gas chromatograph to analyze expired gas samples, following the recommendations of the ATS/ ERS $[15,19]$.

\section{Intrabreath lung diffusing capacity and pulmonary blood flow (qc)}

Pulmonary Blood Flow (Qc) and diffusing capacity of the lungs for carbon monoxide (DLCO) were measured using inert and soluble gases on the CareFusion Vmax system using an intra-breath maneuver [17]. For this maneuver, subjects were asked to breath on a mouthpiece while wearing a nose clip. Subjects were instructed to exhale to residual volume (RV) and then were switched in to an inspiratory reservoir and took a maximal inhalation of a test gas mixture containing $0.3 \%$ carbon monoxide (CO), $0.3 \%$ methane, $0.3 \%$ acetylene, $21 \% \mathrm{O}_{2}$, and balance $\mathrm{N}_{2}$. Subjects were coached to exhale slowly at a steady rate until they were near RV. From the rate of disappearance of $\mathrm{CO}$ and acetylene in comparison to the inert gas methane the rate of disappearance of $\mathrm{CO}$ and acetylene were determined. This rate of disappearance of CO provides the DLCO value. Since acetylene does not bind to hemoglobin the rate of its disappearance is limited only by the flow of blood through the lungs, thereby providing a measure of $\mathrm{Q}[20,21]$.

\section{QOL questionnaires. St. George's respiratory questionnaire}

The SGRQ is a 50-item questionnaire developed to measure health status (quality of life, QOL) in patients with diseases of airways obstruction. Scores are calculated for three domains: Symptoms, Activity and Impacts (Psychosocial) as well as a total score. Psychometric testing has demonstrated its repeatability, reliability and validity. Sensitivity has been demonstrated in clinical trials. A minimum change in score of 4 units has previously been established as clinically relevant. The SGRQ has been used in a range of disease groups including asthma, chronic obstructive pulmonary disease (COPD) and bronchiectasis, and in a range of settings such as randomized controlled therapy trials and population surveys [22]. The SGRQ correlates significantly with other measures of disease activity such as cough, dyspnea, 6-min walk test and $\mathrm{FEV}_{1}$ as well as other measures of general health such as the Sickness Impact Profile (SIP) score which evaluates the impact of disease on physical and emotional functioning and Short Form 36 (SF36) health survey which is a patient reported survey of health [23].

\section{Gas exchange, ventilation and lung mechanics}

During exercise testing oxygen consumption $\left(\mathrm{VO}_{2}\right)$, carbon dioxide production $\left(\mathrm{VCO}_{2}\right)$, breathing frequency $(\mathrm{fb})$, tidal volume $\left(\mathrm{V}_{\mathrm{t}}\right)$, minute ventilation $\left(\mathrm{V}_{\mathrm{E}}\right)$ and derived variables (e.g., $\mathrm{V}_{\mathrm{E}} / \mathrm{VCO}_{2}$ ) were measured continuously or calculated using a low resistance open circuit automated metabolic system (CareFusion).

\section{Statistics}

We were interested in the association of resting measures of DLCO measured via single breath or intrabreath methods as well as expressed relative to Qc with exercise capacity (peak $\mathrm{VO}_{2}$ ) in patients with moderate to severe COPD and if these measures were more highly associated to exercise capacity than more typical measures of lung mechanics. Descriptive statistics were used to describe patient characteristics and demographics while multiple regression and correlational analysis were used to determine associations between DLCO, Q, lung mechanics, QOL, disease severity and exercise capacity. Statistics were performed with a combination of EXCEL and the statistical software package JMP Statistical Discovery TM software from SAS.

\section{Results}

Subject characteristics and pulmonary function measures Thirty two subjects completed the study. As shown in Table 1, on average our study cohort was older, approximately half female, above ideal body mass index and had a 35 pack year smoking history. By design, their GOLD classification ranged from 1 to 4 with an average classification consistent with moderate disease with an $\mathrm{FEV}_{1}$ of $56 \%$ of age predicted and an $\mathrm{FEV}_{1} / \mathrm{FVC}$ ratio of $59 \%$ (Table 2). None of the subjects were on continuous oxygen or oxygen for exercise at the time of the study. The majority of subjects were on combination inhalation therapy that included inhaled beta- 2 agonist, anticholinergic, and inhaled steroid with a minority of subjects on oral steroids. Quality of life scores based from the St George questionnaire was consistent with severity of disease as described by the GOLD classification. 
Table 1 Subject characteristics ( $n=32$ )

\begin{tabular}{lll}
\hline & Mean \pm SD & Range \\
\hline Age (years) & $66 \pm 9$ & $46-84$ \\
\% Female & 47 & - \\
Weight (Kg) & $88 \pm 23$ & $36-155$ \\
BMI (Kg/m2) & $30 \pm 6$ & $13-44$ \\
Smoking history (pack year) & $35 \pm 29$ & $0-120$ \\
Current/former/never smoker (n) & $6 / 22 / 4$ & - \\
GOLD Classification (1-4) & $2.3 \pm 0.8$ & $1-4$ \\
St George Respiratory Questionnaire & $44 \pm 21$ & $8-84$ \\
Inhaled beta agonist (\%) & 97 & - \\
Inhaled anticholinergic (\%) & 59 & - \\
Inhaled steroid (\%) & 68 & - \\
Oral steroid (\%) & 20 & - \\
\hline
\end{tabular}

GOLD Global Initiative for Chronic Obstructive Lung Disease classification for air flow obstruction

\section{Resting lung diffusion measures - Single breath vs intra- breath}

Table 3 lists single breath and intra breath DLCO measures, the measured pulmonary blood flow (Qc), and Hgb values. SBDLCO averaged $13.2 \mathrm{ml} / \mathrm{min} / \mathrm{mmHg}$ and $58 \%$ of predicted with the average IBDLCO $71 \%$ of the SB method ranging from 20 to $110 \%$ across the study population. Overall the SB and IB methods were highly correlated with an $\mathrm{r}$ of 0.88 (Fig. 1) and the IB/SBDLCO relationship was positively associated with resting IC (Fig. 2). The measured pulmonary blood flow (Qc) using soluble gas was $76 \%$ of the resting predicted cardiac output based on gender and body size. On average the SBDLCO was $51 \%$ predicted in current smokers vs $56 \%$ predicted in those that had quit or never smoked. Though the current smokers were slightly reduced relative to nonsmokers, there was no statistical difference between groups $(p<0.05)$.

\section{Cardiopulmonary exercise responses}

Cardiopulmonary exercise responses are reported in Table 4. On average the peak $\mathrm{VO}_{2}$ for the group was $0.98 \mathrm{~L} / \mathrm{min}$ or $11.4 \mathrm{ml} / \mathrm{kg} / \mathrm{min}$ equivalent to $50 \%$ of age

Table 2 Pulmonary Function Variables

\begin{tabular}{lll}
\hline & Mean \pm SD & Percent Predicted (range) \\
\hline FVC $(\mathrm{L})$ & $2.48 \pm 0.69$ & $75 \pm 15$ \\
$\mathrm{FEV}_{1}(\mathrm{~L})$ & $1.51 \pm 0.58$ & $56 \pm 16$ \\
$\mathrm{FEV}_{1} / \mathrm{FVC}$ & $59 \pm 11$ & $(33-78)$ \\
$\mathrm{FEF}_{25-75}(\mathrm{~L} / \mathrm{s})$ & $0.75 \pm 0.38$ & $26 \pm 13$ \\
$\mathrm{FEF}_{75}(\mathrm{~L} / \mathrm{S})$ & $0.29 \pm 0.11$ & $27 \pm 13$ \\
$\mathrm{MW}(\mathrm{L} / \mathrm{m})$ & $48 \pm 19$ & $45 \pm 17$ \\
\hline
\end{tabular}

FVC Forced Vital Capacity, FEV 1 Forced Expiratory Volume in $1 \mathrm{~s}$, FEF Forced Expiratory Flow, MVV Maximal voluntary ventilation. All data are pre bronchodilator and gender predicted. Average peak heart rate was $103 \mathrm{bpm}$ which was $70 \%$ of their age predicted and average respiratory exchange ratio (RER) was 1.03. Inspiratory capacity consistently fell throughout exercise and at peak the tidal volume reached $65 \%$ of the IC. Oxygen pulse rose to an average of $8 \mathrm{ml} /$ beat early in exercise but then plateaued thereafter and reached a max of 9.1 in peak exercise suggesting a plateauing of cardiac stroke volume. Subjects complained of both general fatigue and dyspnea as major reasons for stopping the test. At peak exercise the minute ventilation averaged $73 \%$ of the maximum voluntary ventilation (MVV) with five subjects exceeding their pre-test MVV.

\section{Relationship of resting measures of lung mechanics and lung diffusion to exercise capacity}

Univariate correlations of resting measures of lung function, QOL, anthropometric measures and lung diffusion relative to exercise capacity (expressed in $\mathrm{ml} / \mathrm{kg} / \mathrm{min}$ as well as in $\mathrm{L} / \mathrm{min}$ ) are shown in Table 5 . The values that were most significantly linked to exercise capacity based on $\mathrm{VO}_{2}$ peak in $\mathrm{ml} / \mathrm{kg} / \mathrm{min}$ were SBDLCO relative to pulmonary blood flow (SBDLCO/Qc) (Fig. 3), SBDLCO (\% Pred), VT/IC, (where VT is tidal volume), absolute measures of $\mathrm{FEF}_{25-75}$ (Fig. 4), $\mathrm{FEF}_{75}$ and $\mathrm{BMI}(p<0.01)$. Also associated but less significantly so $(p<0.05$ but $>0.01)$ were the IBDLCO also relative to Qc as well as FVC and FEF50\%. In a step wise fashion or when allowing all significant variables to compete in a multiple regression, only SBDLCO/Qc and BMI remained in a model predicting $\mathrm{VO}_{2}$ peak $\mathrm{ml} / \mathrm{kg} / \mathrm{min}$ where:

$$
\left[\begin{array}{c}
\mathrm{VO}_{2} \text { peak } \mathrm{ml} / \mathrm{kg} / \mathrm{min}=2.51(\mathrm{DLCO} / \mathrm{Qc})-0.176(B M I)+11.48 \\
\text { with an } \mathrm{r} \text { of } 0.73 \text { and an } R^{2} \text { of } 0.53 .
\end{array}\right]
$$

When expressing $\mathrm{VO}_{2}$ peak as $\mathrm{L} / \mathrm{min}, \mathrm{SBDLCO} / \mathrm{Qc}$, $\mathrm{IC}, \mathrm{VT} / \mathrm{IC}$ and BSA were the most predictive of exercise capacity with FVC being the strongest lung mechanics measure in a multiple regression but which lost significance when BSA was added. Thus, the best model for absolute $\mathrm{VO}_{2}$ was:

$\left[\begin{array}{c}V_{2} \text { peak } L / \min =0.2038(D L C O / Q c)+0.6193(B S A)-0.665 \\ \text { with an } r \text { of } 0.81 \text { and an } R^{2} \text { of } 0.65\end{array}\right]$

\section{Influence of smoking and gender on predictors of exercise capacity}

Since six of our cohort were current smokers and current smoking and the time of abstention from smoking is known to impact DLCO [24, 25], we performed both stepwise and multivariate models accounting for smokers. Under both conditions, SBDLCO/Qc and BMI 
Table 3 Lung diffusing capacity and pulmonary blood flow

\begin{tabular}{lll}
\hline & Mean \pm SD & Percent predicted or (Range) \\
\hline Single Breath DLCO (SBDLCO, ml/min/mmHg) & $13.2 \pm 5.5$ & $58 \pm 23(31-112)$ \\
Intra Breath DLCO (IBDLCO, ml/min/mmHg) & $9.7 \pm 5.9$ & $(1.3-27)$ \\
IBDLCO/SBDLCO (\%) & $71 \pm 26$ & $(20-110)$ \\
Pulmonary Blood Flow (Qc, L/m, measured) & $4.8 \pm 0.9$ & $(3.3-6.8)$ \\
Pulmonary Blood Flow -Cardiac output, (L/m, Predicted) & $6.3 \pm 0.4$ & $(5.4-7.1)$ \\
SBDLCO/Qc ratio & $2.8 \pm 1.2$ & $(1.2-5.7)$ \\
Hgb (g/dl) & $13.5 \pm 1.7$ & $(11-17)$ \\
\hline
\end{tabular}

Pulmonary Blood Flow measured with soluble gas method. Cardiac output estimated based on age, gender, BSA, from Ref (William LR). Qc = Pulmonary Blood Flow

still remained significant predictors $(p<0.01)$ with the influence of smoking being not significant $(p=0.67)$. Subgroup analysis excluding current smokers was also performed relative to $\mathrm{VO}_{2}$ peak $\mathrm{ml} / \mathrm{kg} / \mathrm{min}$, where $\mathrm{DLCO} / \mathrm{Q}$ and BMI remained significant predictors $(p=0.000$ and $p=0.009$ respectively). Percent predicted SB and IBDLCO were also not significantly different between current and past smokers $(p>0.05)$.

We also accounted for gender in the models, including when expressing $\mathrm{VO}_{2}$ peak in $\mathrm{L} / \mathrm{min}$ rather than in $\mathrm{ml} /$ $\mathrm{kg} / \mathrm{min}$. There was no influence of gender on the relationship between SBDLCO/Qc and $\mathrm{VO}_{2}$ peak. When expressing $\mathrm{VO}_{2}$ peak in $\mathrm{L} / \mathrm{min}, \mathrm{SBDLCO} / \mathrm{Qc}$ and $\mathrm{BSA}$ remained the most significant predictors.

\section{Discussion}

\section{Primary findings}

From our study we conclude that while resting measures of hyperinflation and maximal expiratory flows,

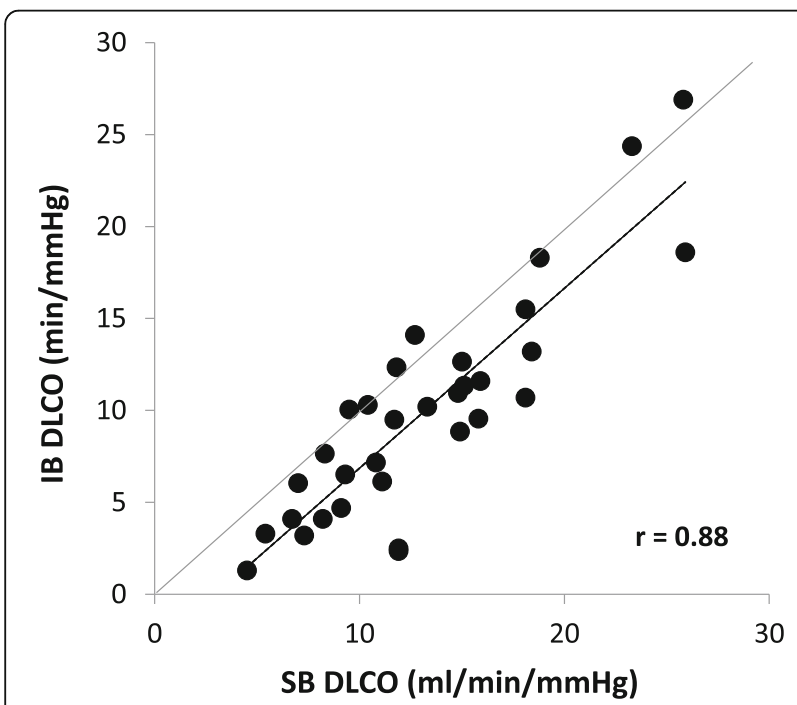

Fig. 1 Relationship of Single Breath DLCO (SBDLCO) to Intra Breath DLCO (IBDLCO, $n=32)$. The IBDLCO was on average lower than the SBDLCO $(p<0.001)$ particularly in patients with values that were more significantly reduced relative to predictive values ( $<65 \%$ of predicted) particularly over the mid to lower lung volumes were predictive of exercise capacity, lung diffusing capacity alone or expressed relative to resting pulmonary blood flow was the most predictive of exercise capacity. Furthermore, when allowed to compete in a multiple regression model, only the SBDLCO relative to Qc and measure of body weight or habitus were significant predictors and explained approximately $50-60 \%$ of the variability in exercise capacity in this population.

\section{Previous studies looking at exercise intolerance in COPD}

Factors contributing to exercise intolerance in patients with COPD are complex. While a number of studies have examined predictors of exercise capacity in the COPD population, the majority of these have focused on measures of lung mechanics and while relationships are found between measures of maximal expiratory flows and volumes, measures of hyperinflation appear to be the most predictive $[3,8,9,26,27]$. In this study we evaluated several measures of lung function, quality of life, body weight/habitus and measures of lung diffusing

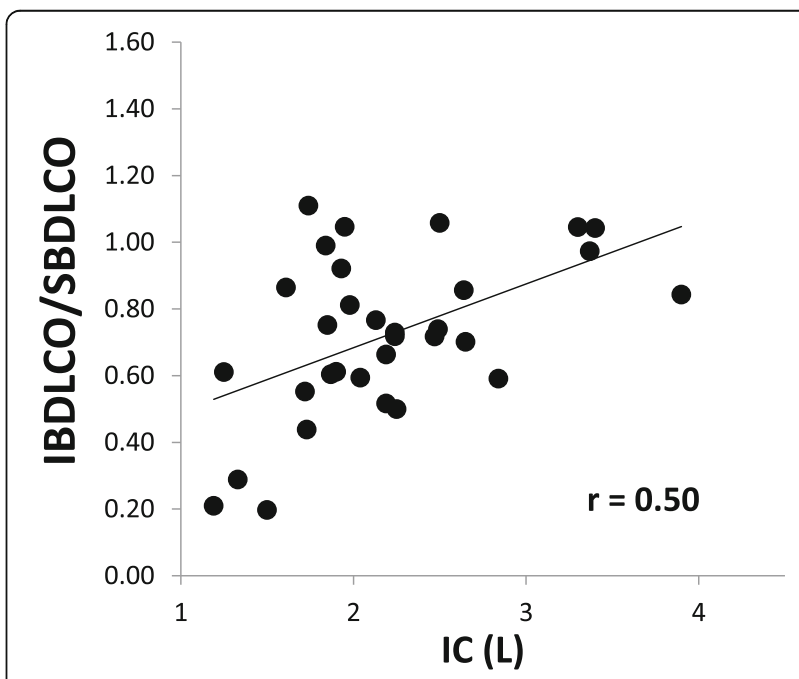

Fig. 2 Relationship of inspiratory capacity (IC) to the IBDLCO and SBDLCO ratio in patients with COPD $(n=32)$. Subjects with the highest IC tended to have the highest ratio of IB to SBDLCO 
Table 4 Breathing pattern, lung mechanics and gas exchange responses to exercise $(n=32)$

\begin{tabular}{llll}
\hline & Rest & First work load & Peak exercise \\
\hline Heart Rate (bpm) & $77 \pm 10$ & $92 \pm 11$ & $103 \pm 22$ \\
RPE (6-20) & $7 \pm 2$ & $11 \pm 2$ & $17 \pm 2$ \\
Dyspnea (0-10) & $1 \pm 1$ & $3 \pm 2$ & $7 \pm 2$ \\
$\mathrm{VE} \mathrm{(L/min)}$ & $12.5 \pm 2.7$ & $24 \pm 6$ & $34 \pm 11$ \\
$\mathrm{Fb} / \mathrm{NT}$ ratio & $26 \pm 15$ & $26 \pm 14$ & $30 \pm 13$ \\
$\mathrm{TI} / \mathrm{TTOT}$ ratio & $39 \pm 8$ & $38 \pm 4$ & $39 \pm 4$ \\
$\mathrm{IC}(\mathrm{L})$ & $2.2 \pm 0.7$ & $1.9 \pm 0.7$ & $1.8 \pm 0.7$ \\
$\mathrm{VT} / \mathrm{IC}(\%)$ & $37 \pm 13$ & $52 \pm 12$ & $65 \pm 27$ \\
$\mathrm{VO}_{2} \mathrm{ml} / \mathrm{kg} / \mathrm{min}$ & $3.7 \pm 0.8$ & $8.3 \pm 1.4$ & $11.4 \pm 3.1$ \\
$\mathrm{VE} / \mathrm{NCO}_{2}$ ratio & $47 \pm 7$ & $37 \pm 5$ & $36 \pm 5$ \\
$\mathrm{PetCO}_{2} \mathrm{mmHg}$ & $35 \pm 5$ & $37 \pm 4$ & $37 \pm 5$ \\
$\mathrm{O}_{2} \mathrm{Pulse}$ & $4 \pm 1$ & $8 \pm 2$ & $9 \pm 3$ \\
$\mathrm{SaO}_{2}(\%)$ & $96 \pm 2$ & $95 \pm 3$ & $94 \pm 3$ \\
\hline VE & &
\end{tabular}

$V E$ Minute ventilation, $f b$ breathing frequency, $V T$ tidal volume, $T /$ inspiratory time, $\Pi T O T$ total respiratory cycle time, $I C$ inspiratory capacity, $V_{2}$ oxygen consumption, $\mathrm{VCO}_{2}$ carbon dioxide production, $\mathrm{PetCO}_{2}$ end tidal partial pressure of carbon dioxide, $\mathrm{O}_{2}$ pulse $\mathrm{VO}_{2}$ /heart rate, $\mathrm{SaO}_{2}$ arterial oxygen saturation estimated from pulse oximetry

Table 5 Univariate Correlations with $\mathrm{VO}_{2}$ peak expressed in $\mathrm{L} / \mathrm{min}$ and $\mathrm{ml} / \mathrm{kg} / \mathrm{min}$

\begin{tabular}{|c|c|c|c|c|}
\hline$N=32$ & $\begin{array}{l}\mathrm{VO}_{2} \text { peak } \\
\mathrm{ml} / \mathrm{kg} / \mathrm{min}\end{array}$ & $p$-value & $\begin{array}{l}\mathrm{VO}_{2} \text { peak } \\
\mathrm{L} / \mathrm{min}\end{array}$ & $p$-value \\
\hline SB DLCO & 0.18 & 0.312 & 0.51 & 0.002 \\
\hline SB DLCO (\%Pred) & 0.49 & 0.004 & 0.48 & 0.006 \\
\hline IB DLCO & 0.20 & 0.277 & 0.51 & 0.002 \\
\hline IB DLCO (\% Pred) & 0.41 & 0.020 & 0.49 & 0.004 \\
\hline $\mathrm{IB} / \mathrm{SB}$ & 0.01 & 0.974 & 0.37 & 0.039 \\
\hline SB DLCO/QC & 0.63 & 0.000 & 0.59 & 0.000 \\
\hline $\mathrm{IC}(\mathrm{L})$ & 0.40 & 0.020 & 0.60 & 0.000 \\
\hline $\mathrm{VT} / \mathrm{IC}$ & -0.42 & 0.017 & -0.55 & 0.001 \\
\hline FVC & 0.41 & 0.018 & 0.54 & 0.001 \\
\hline $\mathrm{FEV}_{1}(\%$ Pred $)$ & 0.38 & 0.030 & 0.22 & 0.217 \\
\hline $\mathrm{FEF}_{25-75}(\mathrm{~L} / \mathrm{min})$ & 0.49 & 0.004 & 0.48 & 0.006 \\
\hline $\mathrm{FEF}_{75}(\mathrm{~L} / \mathrm{min})$ & 0.47 & 0.006 & 0.41 & 0.018 \\
\hline Wt (kg) & -0.30 & 0.092 & 0.55 & 0.001 \\
\hline BMI & -0.42 & 0.017 & 0.32 & 0.078 \\
\hline BSA & 0.16 & 0.370 & 0.64 & 0.000 \\
\hline $\mathrm{QOL}$ & -0.23 & 0.200 & -0.05 & 0.773 \\
\hline GOLD classification & -0.27 & 0.140 & -0.20 & 0.275 \\
\hline
\end{tabular}

SB single breath method, IB intra-breath method. Values in columns 2 through 5 that are most significantly linked to exercise capacity based on $\mathrm{VO}_{2}$ peak are in bold and italics. In column 1, only the variables that predict $\mathrm{VO}_{2}$ peak in $\mathrm{ml} /$ $\mathrm{kg} / \mathrm{min}$ in a multiple regression analysis, are in bold.

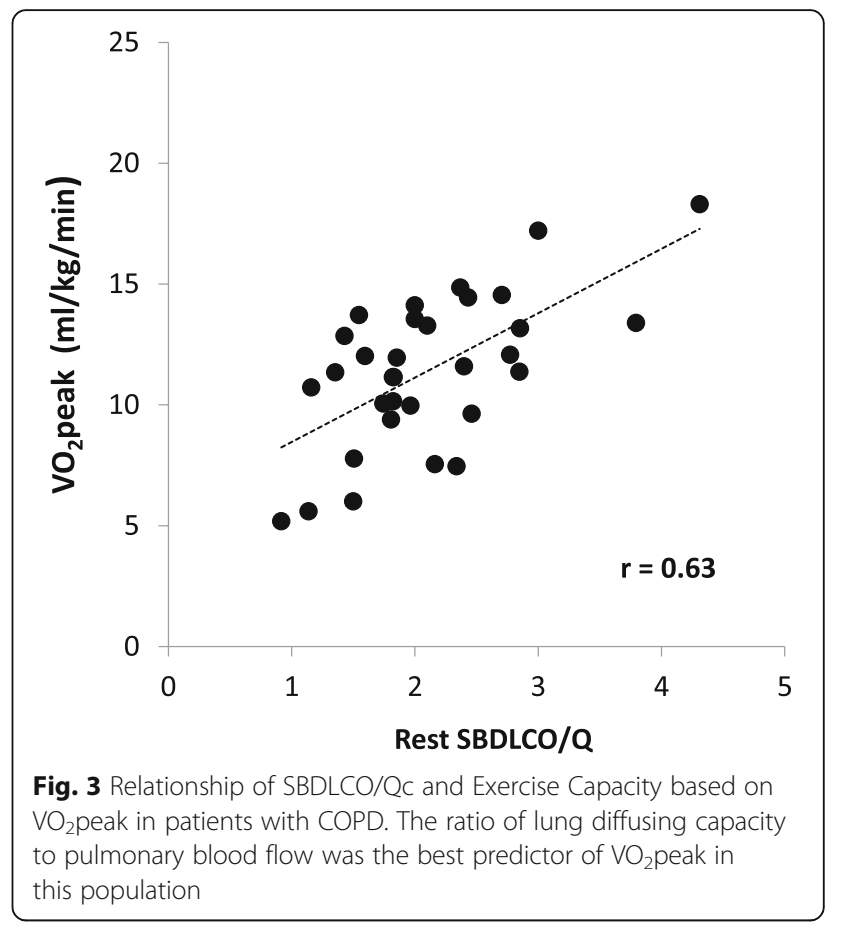

capacity measured differently or expressed relative to lung function and pulmonary blood flow. We found that lung mechanics, particularly flows at the mid to lower lung volumes and the inspiratory capacity relationship, or tidal volume inspiratory capacity relationship, seemed to be the most predictive. However when allowed to compete in a model with measures of lung diffusion and measures of body weight or habitus, the measures of

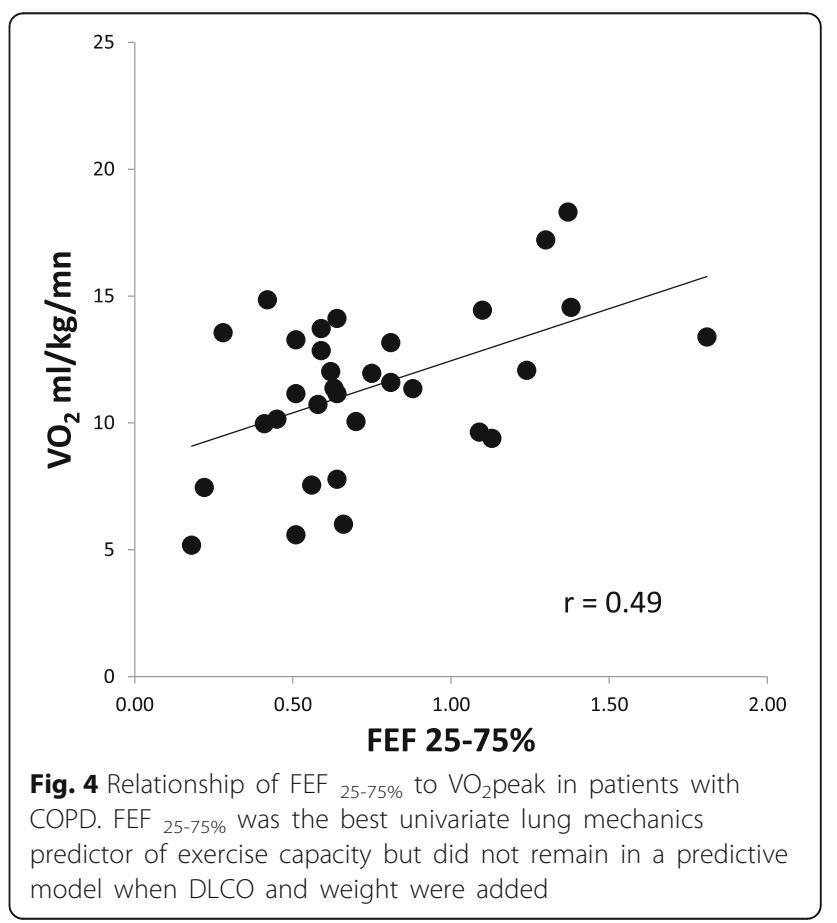


mechanics no longer reached significance. In particular when SBDLCO was expressed relative to the measured Qc with BMI or when predicting $\mathrm{VO}_{2}$ in $\mathrm{L} / \mathrm{min}$, BSA together in a model the mechanics measures, were no longer contributory.

DLCO is a variable of paramount importance in pulmonary medicine. It represents a complex integration of factors including ventilation distribution, matching of ventilation to perfusion, the resistance at the alveolar-capillary membrane as well as the combination rates with hemoglobin. Since all of the above factors can be affected with more classic patterns of emphysema and COPD with ultimate destruction of the alveolar-capillary bed, preservation of DLCO is an important marker of lung health [13]. For example, a major factor contributing to recruitment or distension of the pulmonary capillary bed is cardiac output or pulmonary blood flow (Qc) [28]. As alveolar-capillary walls are remodeled, destroyed or even stiffened with disease, the DLCO/Qc relationship would be altered. With exercise, ventilation rises and pulmonary blood flow increases resulting in elevation of DLCO. In emphysema and COPD with loss of alveolar volume, an important adaption to maintain gas exchange in the face of increased blood cell transit time is a rise in pulmonary capillary blood volume. Thus preservation of this relationship in this population should be a discernible advantage. It was interesting that the IBDLCO, while highly correlated to the SBDLCO, was not as predictive of exercise capacity as the SB method. Our original rational was that since the IB method was performed more quickly and at a lung volume more specific to tidal breathing, that it may be a more sensitive predictor of functional gas exchange surface area. However, during the rapid inspiratory phase of the SBDLCO method, potentially increasing pulmonary blood volume, and with the inhalation to total lung capacity, increasing alveolar volume, it is likely this gives a better overall representation of functional or possibly recruitable surface area available for use during exercise. Also of note was the fact that the IBDLCO method was more variable across subjects and less reproducible than the SB method.

\section{Other predictors of exercise capacity in COPD}

In what have become classical studies by O'Donnell and colleagues, resting IC, degree of hyperinflation with exercise, and change in IC, have all been highly predictive of exercise capacity [9]. Hyperinflation is associated with expiratory flow limitation, volume constraints and less optimal respiratory muscle performance $[3,12,26]$. Dynamic hyperinflation during exercise contributes to perceived respiratory discomfort. Indirect evidence of the importance of dynamic hyperinflation comes from studies that have demonstrated that alleviation of dyspnea following bronchodilator therapy and lung volume reduction surgery (LVRS) are both explained, in part, by reduced operating lung volumes [29]. Additional studies have suggested that COPD patients enter into a spiral of decline associated with reduced activity, inflammation and skeletal muscle dysfunction [2, 30, 31]. A high work and cost of breathing in the setting of elevated operational lung volumes and in some cases excessive expiratory muscle work and also diaphragmatic fatigue contribute to exercise intolerance [30]. COPD also is associated other co-morbidities such as coronary artery disease, pulmonary vascular disease, pulmonary hypertension and right ventricular failure; all of which impair cardiac output and thus compromise oxygen delivery [31]. While the reality is that these collective contributors to exercise limitation in COPD are all integrated and codependent, our work suggests that maintenance of a functional alveolar-capillary bed is an important determinant of patients ability to exercise and likely to carry on normal daily activities.

Targeting the airways and the inflammatory pathways has been the cornerstone of therapy for COPD and emphysema which are accomplished by classes of beta- 2 agonists, anticholinergics, and steroids. Using the same rational, targeting diffusing capacity, i.e. the pulmonary vasculature, by medications has been tried. However, the results have been disappointing. For example, sildenafil, a phosphodiesterase type-5 (PDE-5) inhibitor with vasodilatory properties, commonly used in treatment of pulmonary arterial hypertension (PAH), has been tried in COPD patients who did not have pulmonary hypertension. Interestingly, the drug worsened gas exchange, increased the alveolar-arterial oxygen difference, and did not improve exercise capacity, possibly by causing ventilation-perfusion mismatch, indicating the need for more studies and new medications [26].

\section{Limitations}

There are several limitations relative to this study to consider. First, this was a relatively small study in a somewhat heterogeneous group of primarily moderate to moderately-severe COPD patients who performed cycle ergometry to volitional exhaustion. We essentially recruited consecutive patients with a history of COPD, on stable medications, willing to participate with minimal exclusion criteria. To some extent this was by design so that the study population represents a typical mixed tertiary outpatient population. Lung diffusion has been shown to be predictive of exercise capacity in a number of chronic lung and heart conditions and therefore appears to be a good, more generic marker to consider for the COPD population. Larger studies would however be needed to tease out in which specific COPD populations it may be most predictive. Secondly, we used volitional fatigue as a cessation criteria. This resulted in some subjects with lower than typical RER 
values or other more traditional measures associated with maximal exercise, e.g., heart rate. Cycle ergometry is known to be associated with more local muscle fatigue and may underestimate true maximal exercise. However, we attempted to use similar stopping criteria for our subjects and the same study staff performed all exercise testing which likely resulted in a more uniform representation of peak exercise capacity across subjects. In addition, since mechanical constraint to breathing occurs, some subjects would be unable to hyperventilate to more typical RER values or get to a true cardiovascular limitation. Thus we feel our data are representative of the typical tertiary center testing laboratory where symptom limitation is typically used for stopping criteria.

We also allowed recruitment of current smokers. While subjects were asked not to smoke within $24 \mathrm{~h}$ of testing, we did not specifically assess carboxyhemoglobin levels and therefore could not confirm if they were smoking prior to testing. This could have influenced our DLCO measures, though previously Oglivie et al. felt that the effects of increasing $\mathrm{COHb}$ were sufficiently small, so that routine correction of DLCO was not necessary [32]. As a result, DLCO was not until more recently clinically adjusted for increases in $\mathrm{COHb}$ [33]. We also did not note a difference in percent predicted DLCO between smokers and non-smokers and did not find that current smoking impacted our predictive models. Finally, we used a soluble gas method for calculation of pulmonary blood flow or in the absence of significant shunt or cardiac output. We acknowledge this method may be somewhat dependent on ventilation and perfusion matching in the lungs and therefore may also underestimate actual values.

\section{Conclusions}

In conclusion, exercise limitation in COPD is affected by alveolar-capillary gas exchange impairment which in turn is attributed to impairment of pulmonary circulation. SBDLCO relative to Qc and body weight are better predictors of exercise performance compared to IBDLCO and other respiratory variables in this population. We proposed that lung diffusing capacity, either alone or relative to pulmonary blood flow, is a good measure of pulmonary vascular health in the COPD population and is also a good measure for assessing mechanisms of exercise intolerance in this population. Medical management targeting the pulmonary circulation may help reduce symptoms and improve exercise tolerance in COPD patients.

\section{Abbreviations}

BMl: Body mass index; COPD: Chronic obstructive pulmonary disease DLCO: Diffusing capacity for carbon monoxide; IB: Intrabreath; Qc: Pulmonary blood flow; SB: Single breath; SGQOL: St George quality of life; $\mathrm{VO}_{2}$ : Oxygen consumption

\section{Acknowledgements}

Authors thank David Sinks and Beth Anke from CareFusion for their technical support; and also Salome Illkhan, Angie Holland and other staff in the Cardiopulmonary Exercise lab in Augusta for their assistance in testing and scheduling the patients.

\section{Funding}

None to declare.

Availability of data and materials

The dataset is available for review upon request at the site of study.

\section{Authors' contributions}

MB protocol design, testing subjects, obtaining consent, drafting manuscript; CW protocol design, obtaining consent; AA protocol design; BJ protocol design, statistical analysis. All authors read and approved the final manuscript.

\section{Ethics approval and consent to participate}

The study, ethics, and consent forms were reviewed and approved by the Western Institutional Review Board (WIRB, Pyallup, WA, study number 1153374). Each patient voluntarily participated in the study. If willing to participate, patients signed informed consent.

\section{Consent for publication}

Not applicable.

\section{Competing interests}

The authors declare that they have no competing interest.

\section{Publisher's Note}

Springer Nature remains neutral with regard to jurisdictional claims in published maps and institutional affiliations.

\section{Author details}

${ }^{1}$ University of Central Florida School of Medicine and Division of Critical Care, Florida Hospital, Orlando, FL, USA. ${ }^{2}$ Division of Cardiovascular Diseases, Mayo Clinic, Scottsdale, AZ, USA. ${ }^{3}$ Faculty of Medicine and Health Sciences, Macquarie University, Sydney, NSW, Australia. ${ }^{4}$ PO Box 953814, Lake Mary, FL 32795, USA

Received: 25 July 2016 Accepted: 2 August 2017

Published online: 25 August 2017

References

1. Salzman SH. Which pulmonary function tests best differentiate between COPD phenotypes? Respir Care 2012:57(1):50-7: discussion 8-60.

2. Aliverti A, Macklem PT. The major limitation to exercise performance in COPD is inadequate energy supply to the respiratory and locomotor muscles. J Appl Physiol (1985). 2008;105(2):749-51. discussion 55-7

3. O'Donnell DE. Ventilatory limitations in chronic obstructive pulmonary disease. Med Sci Sports Exerc. 2001;33(7 Suppl):S647-55.

4. Fabbri LM, Romagnoli M, Corbetta L, Casoni G, Busljetic K, Turato G, et al. Differences in airway inflammation in patients with fixed airflow obstruction due to asthma or chronic obstructive pulmonary disease. Am J Respir Crit Care Med. 2003;167(3):418-24.

5. Vogiatzis I, Zakynthinos G, Andrianopoulos V. Mechanisms of physical activity limitation in chronic lung diseases. Pulm Med. 2012;2012:634761.

6. Johnson BD, Badr MS, Dempsey JA. Impact of the aging pulmonary system on the response to exercise. Clin Chest Med. 1994:15(2):229-46.

7. Hyatt RE. Expiratory flow limitation. J Appl Physiol Respir Environ Exerc Physiol. 1983:55(1 Pt 1):1-7.

8. O'Donnell DE, Bertley JC, Chau LK, Webb KA. Qualitative aspects of exertional breathlessness in chronic airflow limitation: pathophysiologic mechanisms. Am J Respir Crit Care Med. 1997:155(1):109-15.

9. O'Donnell DE, Revill SM, Webb KA. Dynamic hyperinflation and exercise intolerance in chronic obstructive pulmonary disease. Am J Respir Crit Care Med. 2001;164(5):770-7.

10. Gallagher CG. Exercise limitation and clinical exercise testing in chronic obstructive pulmonary disease. Clin Chest Med. 1994;15(2):305-26. 
11. Gelb AF, Zamel N, McKenna RJ Jr, Brenner M. Mechanism of short-term improvement in lung function after emphysema resection. Am J Respir Crit Care Med. 1996;154(4 Pt 1):945-51.

12. Martinez FJ, de Oca MM, Whyte RI, Stetz J, Gay SE, Celli BR. Lung-volume reduction improves dyspnea, dynamic hyperinflation, and respiratory muscle function. Am J Respir Crit Care Med. 1997;155(6):1984-90.

13. Farkhooy A, Janson C, Arnardottir RH, Malinovschi A, Emtner M, Hedenstrom H. Impaired carbon monoxide diffusing capacity is the strongest predictor of exercise intolerance in COPD. COPD. 2013;10(2):180-5.

14. Snyder EM, Johnson BD, Beck KC. An open-circuit method for determining lung diffusing capacity during exercise: comparison to rebreathe. J Appl Physiol (1985). 2005;99(5):1985-91.

15. Macintyre N, Crapo RO, Viegi G, Johnson DC, van der Grinten CP, Brusasco $V$, et al. Standardisation of the single-breath determination of carbon monoxide uptake in the lung. Eur Respir J. 2005:26(4):720-35.

16. Huang YC, Helms MJ, Maclntyre NR. Normal values for single exhalation diffusing capacity and pulmonary capillary blood flow in sitting, supine positions, and during mild exercise. Chest. 1994;105(2):501-8.

17. Huang YC, O'Brien SR, MacIntyre NR. Intrabreath diffusing capacity of the lung in healthy individuals at rest and during exercise. Chest. 2002;122(1):177-85.

18. Mador MJ, Rodis A, Magalang UJ. Reproducibility of Borg scale measurements of dyspnea during exercise in patients with COPD. Chest. 1995;107(6):1590-7.

19. Brusasco V, Crapo R, Viegi G, American Thoracic S, European Respiratory S. Coming together: the ATS/ERS consensus on clinical pulmonary function testing. Eur Respir J. 2005;26(1):1-2.

20. Charloux A, Enache I, Richard R, Oswald-Mammosser M, Lonsdorfer-Wolf E, Piquard $\mathrm{F}$, et al. Diffusing capacity of the lung for $\mathrm{CO}$ and pulmonary blood flow during incremental and intermittent exercise. Scand J Med Sci Sports. 2010;20(1):e121-9.

21. Martonen TB, Wilson AF. Theoretical basis of single breath gas absorption tests. J Math Biol. 1982;14(2):203-20.

22. Jones PW, Quirk FH, Baveystock CM. The St George's respiratory questionnaire. Respir Med. 1991;85(Suppl B):25-31. discussion 3-7

23. Bergner M, Bobbitt RA, Kressel S, Pollard WE, Gilson BS, Morris JR. The sickness impact profile: conceptual formulation and methodology for the development of a health status measure. Int J Health Serv. 1976;6(3):393-415.

24. Graham BL, Mink JT, Cotton DJ. Effects of increasing carboxyhemoglobin on the single breath carbon monoxide diffusing capacity. Am J Respir Crit Care Med. 2002;165(11):1504-10.

25. Sansores RH, Pare PD, Abboud RT. Acute effect of cigarette smoking on the carbon monoxide diffusing capacity of the lung. Am Rev Respir Dis. 1992; 146(4):951-8.

26. O'Donnell DE, Lam M, Webb KA. Measurement of symptoms, lung hyperinflation, and endurance during exercise in chronic obstructive pulmonary disease. Am J Respir Crit Care Med. 1998;158(5 Pt 1):1557-65.

27. Johnson BD, Weisman IM, Zeballos RJ, Beck KC. Emerging concepts in the evaluation of ventilatory limitation during exercise: the exercise tidal flowvolume loop. Chest. 1999;116(2):488-503.

28. Williams LR. Reference values for total blood volume and cardiac output in humans. US Dept of Energy, Oakridge National Laboratory, Health Sciences Research Division; 1994. ORNL/TM-12814:1-51. https://www.osti.gov/scitech/ biblio/10186900

29. Belman MJ, Botnick WC, Shin JW. Inhaled bronchodilators reduce dynamic hyperinflation during exercise in patients with chronic obstructive pulmonary disease. Am J Respir Crit Care Med. 1996;153(3):967-75.

30. Mador MJ, Kufel TJ, Pineda LA, Sharma GK. Diaphragmatic fatigue and highintensity exercise in patients with chronic obstructive pulmonary disease. Am J Respir Crit Care Med. 2000;161(1):118-23.

31. Morrison DA, Adcock K, Collins CM, Goldman S, Caldwell JH, Schwarz MI. Right ventricular dysfunction and the exercise limitation of chronic obstructive pulmonary disease. J Am Coll Cardiol. 1987;9(6):1219-29.

32. Ogilvie CM, Blakemore WS, Forster RE, Morton JW. A standardized breath holding technique for the clinical measurement of the diffusing capacity of the lung for carbon monoxide. J Clin Invest. 1957;36(1 Part 1):1-17.

33. Viegi G, Paoletti P, Prediletto R, Di Pede F, Carrozzi L, Carmignani G, et al. Carbon monoxide diffusing capacity, other indices of lung function, and respiratory symptoms in a general population sample. Am Rev Respir Dis. 1990;141(4 Pt 1):1033-9. 